\title{
Efeitos do Transtorno de Estresse Pós-Traumático na Memória
}

\author{
Nathalia Balloni Emygdio ${ }^{1}$ \\ ${ }^{1}$ Universidade Presbiteriana Mackenzie, SP, Brasil. \\ Adriana Cristine Fonseca Mozzambani² \\ ${ }^{2}$ Universidade Federal de São Paulo, SP, Brasil. \\ Camila Cruz Rodrigues ${ }^{1}$ \\ ${ }^{1}$ Universidade Presbiteriana Mackenzie, SP, Brasil.
}

\author{
Simone Freitas Fuso ${ }^{1}$ \\ ${ }^{1}$ Universidade Presbiteriana Mackenzie, SP, Brasil. \\ Natália Alves Acedo ${ }^{1}$ \\ ${ }^{1}$ Universidade Presbiteriana Mackenzie, SP, Brasil. \\ Marcelo Feijó de Mello ${ }^{2}$ \\ ${ }^{2}$ Universidade Federal de São Paulo, SP, Brasil.
}

Resumo: OTranstorno de Estresse Pós-Traumático (TEPT) é frequentemente associado a déficits cognitivos, porém ainda existem divergências com relação as funções cognitivas afetadas. $\mathrm{O}$ objetivo do presente estudo foi avaliar o impacto do TEPT na memória operacional, na memória visual de curto prazo, na memória episódica de longo prazo, na memória semântica de longo prazo e na memória prospectiva. A amostra foi composta por 20 mulheres com idades entre $20 \mathrm{e}$ 60 anos, sendo 10 mulheres provenientes do Programa de Atendimento e Pesquisa em Violência da Universidade Federal de São Paulo (PROVE) com diagnóstico deTEPT (GTEPT) e 10 mulheres controle com a mesma idade e escolaridade (GC). O instrumento utilizado foi o Instrumento de Avaliação Neuropsicológica Breve (NEUPSILIN). Os dados foram analisados por teste t de Student para amostras dependentes e o nível de significância adotado foi de 5\%. Os resultados obtidos a partir da análise do NEUPSILIN apontam para prejuízos na memória operacional ( $\mathrm{p}$ $=0,04)$ e na memória prospectiva $(\mathrm{p}=0,02)$ associados ao TEPT. Porém não foram observadas diferenças entre os grupos no que diz respeito aos outros tipos de memória investigados ( $p$ $>0,05$ ). Os achados deste estudo, com relação aos prejuízos na memória operacional e na memória prospectiva associados ao TEPT estão de acordo com a literatura. Ambas funções são de extrema importância para o desempenho de atividades cotidianas, portanto, a intervenção e reabilitação destas funções no sujeito com TEPT podem auxiliar significativamente na melhora da qualidade de vida.

Palavras-chave: Memória, TEPT Complexo, Violência, Mulheres. 


\title{
Effects of Post Traumatic Stress Disorder in Memory
}

\begin{abstract}
Posttraumatic Stress Disorder (PTSD) is often associated with cognitive deficits, but there are still some differences regarding cognitive functions affected. Therefore, the aim of this study was to evaluate the impact of PTSD on working memory, visual shortterm memory, long-term episodic memory, long-term semantic memory and prospective memory. The sample consisted of 20 women aged between 20 and 60 years, ten women from the Program of Assistance and Research on Violence of the Federal University of São Paulo (PROVE) diagnosed with PTSD and overlapping violence (GTEPT) and ten women control of the same age and educational level (GC). The instrument used was the Brief Neuropsychological Assessment Instrument (NEUPSILIN). Data were analyzed by Student's t-test for dependent samples, and the significance level was $5 \%$. The results obtained from the analysis NEUPSILIN point to the losses in working memory $(\mathrm{p}=0.04)$ and prospective memory $(p=0.02$ ) associated with PTSD. Differences between groups were not observed on other types of memory investigated $(p>0.05)$. The findings of this study on losses in working memory and prospective memory associated with PTSD are in agreement with the literature. Both functions are critical to the performance of daily activities. Therefore, intervention and rehabilitation of these functions in the subject with PTSD can help significantly in improving the quality of life.
\end{abstract}

Keywords: Memory, Complex PTSD, Violence, Women.

\section{Efectos del Trastorno de Estrés Postraumático en la Memoria}

Resumen: El trastorno de estrés postraumático (TEPT) suele asociarse a déficits cognitivos, pero todavía existen divergencias con las funciones cognitivas afectadas. El objetivo del presente estudio fue evaluar el impacto del TEPT en la memoria operativa, en la memoria visual de corto plazo, en la memoria episódica de largo plazo, en la memoria semántica de largo plazo y en la memoria prospectiva. La muestra fue compuesta por 20 mujeres con edades entre 20 y 60 años, siendo 10 mujeres provenientes del Programa de Atención e Investigación en Violencia de la Universidad Federal de São Paulo (PROVE) con diagnóstico de TEPT (GTEPT) y 10 mujeres control con la misma edad y escolaridad (GC). El instrumento utilizado fue el Instrumento de Evaluación Neuropsicológica Breve (NEUPSILIN). Los datos fueron analizados por test $t$ de Student para muestras dependientes y el nivel de significancia adoptado fue del $5 \%$. Los resultados obtenidos a partir del análisis del NEUPSILIN apuntan a pérdidas en la memoria operativa $(p=0,04)$ y en la memoria prospectiva $(p=0,02)$ asociados al TEPT. Sin embargo, no se observaron diferencias entre los grupos en lo que se refiere a los otros tipos de memoria investigados $(p>0,05)$. Los hallazgos de este estudio, con relación a los perjuicios en la memoria operativa y en la memoria prospectiva asociados al TEPT están de acuerdo con la literatura. Ambas funciones son de extrema importancia para el desempeño de actividades cotidianas, por lo tanto, la intervención y rehabilitación de estas funciones en el sujeto con TEPT pueden auxiliar significativamente en la mejora de la calidad de vida.

Palabras clave: Memoria, TEPT Complejo, Violencia, Mujeres. 


\section{Introdução}

De acordo com o estudo realizado pelo Instituto de Pesquisa Econômica Aplicada (IPEA) ocorrem por ano cerca de 5.664 mortes de mulheres vítimas de violência, 472 mortes por mês, 15,52 mortes por dia ou uma morte por cada uma hora e meia (Garcia, 2013). A última pesquisa realizada pelo DataSenado sobre violência doméstica e familiar mostrou que uma em cada cinco brasileiras já sofreram algum tipo de violência, seja ela doméstica ou familiar. E apresentou também que $100 \%$ das mulheres entrevistadas conhecem a Lei Maria da Penha (Lopes et al., 2015).

Segundo o levantamento realizado pela Central de Atendimento à Mulher, no ano de 2014 ocorreram 52.957 denúncias de violência contra a mulher, sendo que destas denúncias, 27.369 são de violência física $(51,68 \%), 16.846$ de violência psicológica (31,81\%), 5.126 de violência moral $(9,68 \%), 1.028$ de violência patrimonial (1,94\%), 1.517 de violência sexual (2,86\%), 931 de cárcere privado (1,76\%) e 140 envolvendo tráfico $(0,26 \%)$. Este levantamento também apontou que em $42,93 \%$ dos casos denunciados ocorrem violência diária e em $34,05 \%$ a violência ocorre com frequência semanal, o que aponta que cerca de $77 \%$ das mulheres sofrem violência com alta frequência (Menicucci, 2014).

No ano de 2011 no Brasil, foram registrados 12.087 casos de estupro, segundo um levantamento realizado pelo IPEA (Cerqueira, \& Coelho, 2014).

Segundo o Mapa de Violência, no Brasil entre os anos de 1980 e 2012 ocorreram 880.386 mortes por arma de fogo. O número de mortes por arma de fogo no Brasil aumentou 387\% de 1980 a 2012 (Waiselfisz, 2015). Por conta disso, a violência é um assunto que merece atenção por parte dos órgãos governamentais, da comunidade científica e da sociedade em geral.

De acordo com McEwen (2003), ao correr perigo o ser humano reage ou enfrentando o adversário ou fugindo deste, de qualquer modo, ele reage com estresse, passando a energia das necessidades em longo prazo para as necessidades imediatas, secretando o hormônio e neurotransmissor adrenalina para auxiliar na resposta de lutar ou fugir.

A amígdala cerebelar tem um papel muito importante na reação ao estresse, pois ela fica responsável pela resposta emocional, portanto, ela está diretamente relacionada com os mecanismos de luta ou fuga. O estímulo estressor é processado primeiramente pela amígdala, que fica responsável pela rea- ção emocional imediata, somente após a reação ao estresse está informação é processada pelo córtex pré-frontal, que neste caso, fica responsável por conter os alarmes instintivos acionados pela amígdala cerebelar (McEwen, 2003).

Quando o sujeito entra nesse mecanismo de luta ou fuga, ocorrem uma série de modificações em seu organismo, dentre elas estão, a aceleração do pulso, o aumento da função cardiorrespiratória, a dilatação dos pulmões, o aumento da oxigenação do cérebro, a contração dos vasos sanguíneos, a liberação das reservas de energia, a liberação de endorfina para função analgésica, a liberação de fibrinogênio para aceleração da coagulação sanguínea, a diminuição da atividade gastrointestinal e a diminuição da função reprodutora (McEwen, 2003).

Ao se expor a um evento traumático o sistema nervoso central, o sistema nervoso periférico, o sistema neuroendócrino e o sistema imunológico são ativados (Glaser, 2000). O eixo hipotálamo-pituitária-adrenal (HPA) fica responsável pelo ajustamento da reação ao estresse, liberando o hormônio cortisol (Sapolsky, Romero, \& Munck, 2000).

Essas modificações no organismo do sujeito são necessárias para a sobrevivência, todavia o estresse crônico pode causar sérios danos ao sistema imunológico, ocasionando uma maior susceptibilidade para ao desenvolvimento de resfriados, infecções, alergias, asma e estados autoimunes. O estresse intenso também está relacionado com o desenvolvimento de doenças cardiovasculares (McEwen, 2003).

De acordo com Peres e Nasello (2005), os sujeitos com Transtorno de Estresse Pós-Traumático (TEPT) têm demonstrado uma resposta exagerada da amígdala somada a uma diminuição da atividade do córtex pré-frontal, que não consegue conter a resposta exagerada ao estresse.

Algumas pesquisas garantem uma hipofunção do eixo HPA e baixos níveis de cortisol associada ao TEPT, portanto, o ajustamento da reação ao estresse fica comprometido (Graeff, 2003), embora hoje não há consenso sobre a direção das alterações deste hormônio (Borges, 2007). Segundo McEwen (2003), o estresse traumático também está relacionado à atrofia de partes do cérebro.

Segundo a $5^{\mathrm{a}}$ edição do Manual Diagnóstico e Estatístico de Transtornos Mentais - DSM 5 (American Psychiatric Association, 2014), o TEPT é um transtorno que afeta adultos, adolescente e crianças. 
Envolve a participação em um evento violento e traumático, onde o sujeito pode ter participação direta, quando o sujeito é a própria vítima; ou indireta, no caso de testemunhar a situação violenta, ou saber sobre um evento traumático que ocorreu com algum familiar ou amigo próximo, ou ser exposto de forma repetida à detalhes de um evento traumático, como no caso de socorristas.

Os sujeitos com este transtorno têm sintomas intrusivos associados ao trauma, no qual os sujeitos revivesciam a situação traumática por meio de sonhos, lembranças e reações dissociativas. Outro sintoma característico do TEPT é a evitação persistente de estímulos associados ao evento traumático. Ocorrem também alterações negativas no humor e na cognição, com sentimentos de distanciamento, incapacidade de recordar detalhes do evento estressor, crenças negativas em relação a si e aos outros, estado emocional negativo persistente, interesse diminuído em atividades significativas e incapacidade persistente de sentir emoções positivas. Somado a estes sintomas também ocorrem alterações na excitabilidade, levando o sujeito a ter perturbações do sono, hipervigilância, surtos de raiva, comportamento imprudente, problemas de concentração e resposta de sobressalto exagerada. Todos os sintomas descritos acima devem ter duração superior a um mês e causar prejuízos significativos no âmbito profissional e social do sujeito (American Psychiatric Association, 2014)

Segundo Buckley, Blanchard e Neill (2000), a sobreposição de eventos negativos pode ser um fator de risco para o desenvolvimento do TEPT. Em contrapartida, de acordo com Heller, Larrieu, D'imperio e Boris (1999), o suporte social seria um fator de proteção ao desenvolvimento do TEPT, o que ressalta a importância da procura a atendimentos de acolhimento após um evento violento, para que se possa prevenir o desenvolvimento do transtorno.

Sujeitos que passaram por múltiplas e prolongadas experiências traumáticas podem desenvolver um quadro mais complexo do que o quadro comum de TEPT. Alguns autores têm usado os termos TEPT complexo ou trauma complexo para nomear estes casos (Herman, 1992).

No TEPT complexo há uma alta taxa de comorbidade com outros transtornos psiquiátricos, como transtorno do pânico, depressão maior, transtorno de ansiedade generalizada e abuso de substâncias
(Cloitre, Cohen, Edelman, \& Han, 2001; Roestel, \& Kersting, 2008).

Segundo Herman e Harvey (1997), além de todos os sintomas característicos do TEPT, estes sujeitos também podem apresentar outros tipos de sintomas, como sintomas somáticos, dissociativos e afetivos. Dentre os sintomas somáticos, podem ocorrer dores de cabeça, dores abdominais, dores lombares, dores pélvicas, tremores, sensação de choque, náusea e distúrbios gastrointestinais. Dentre os sintomas dissociativos, podem ocorrer alterações de personalidade, alterações na realidade, confusão temporal e alucinações. Dentre os sintomas afetivos, podem ocorrer apatia, insônia, sintomas depressivos, dificuldades de concentração, comportamentos suicidas e alterações na percepção sobre si e sobre o mundo).

Estudos apontam prejuízos tanto funcionais quanto estruturais relacionados ao TEPT (Horner, \& Hamner, 2002). A partir de estudos de neuroimagem pode-se observar alterações no volume do hipocampo, na amígdala, no giro cingulado anterior, no córtex pré-frontal (Wignall et al., 2004) e alterações no hipotálamo (Horner, \& Hamner, 2002; Wignall et al., 2004). Essas regiões são responsáveis pela aprendizagem, memória, atenção, controle executivo e regulação emocional (Horner, \& Hamner, 2002). Nesse sentido, o TEPT e as alterações estruturais e funcionais dos sistemas neurobiológicos do estresse podem ser entendidas como um ajustamento do cérebro a um contexto de desenvolvimento violento (Borges, 2007).

Pesquisas corroboram que há déficits cognitivos associados ao TEPT, como por exemplo, prejuízo das funções executivas (Koso, \& Hansen, 2006; Kristensen, 2005; Stein, Kennedy, \& Twamley, 2002), das habilidades viso-construtivas (Stein et al., 2002), da atenção (Koso, \& Hansen, 2006; Stein et al., 2002), do processamento emocional (Kristensen, 2005), da flexibilidade mental e da velocidade psicomotora (Stein et al., 2002).

Entre os déficits de memória apresentam-se prejuízos na memória operacional, na memória declarativa (Koso, \& Hansen, 2006; Stein et al., 2002) e na memória prospectiva (Moradi, Doost, Taghavi, Yule, \& Dalgleish, 1999). E dentre os déficits de atenção apresentam-se prejuízos na atenção seletiva (Kristensen, 2005) e na atenção sustentada (Stein et al., 2002).

Porém há resultados divergentes sobre alterações nas funções cognitivas associadas ao TEPT. Nos estudos de Borges (2007) não foram encontrados prejuízos 
na atenção, na memória, na aprendizagem verbal, na velocidade psicomotora, na flexibilidade mental, na memória operacional e na memória declarativa. No mesmo sentido, na pesquisa de Pederson et al. (2004), não foram observados prejuízos de memória declarativa e redução do volume do hipocampo em pacientes com TEPT.

Levando-se em consideração que a memória não é um sistema unitário e que existem diferentes tipos de memória torna-se necessário compreender em quais tipos de memória e em qual etapa o TEPT poderia influenciar.

O processamento de informações na memória passa por três estágios: codificação, armazenamento e evocação. No estágio de codificação ocorre a aquisição da informação, logo em seguida, esta informação é armazenada na memória e fica disponível para o processo de evocação, no qual ocorre a recuperação da informação armazenada (Baddeley, 2000).

Atkinson e Shiffrin (1968) dividem o processo de memória em memória sensorial, memória de curto prazo e memória de longo prazo. Assim, as informações são recebidas primeiramente no armazenamento da memória sensorial, onde as informações se mantêm por segundos ou frações de segundos após a apresentação de um estímulo. Depois, estas informações passam para o armazenamento na memória de curto prazo, no qual apenas uma quantidade de informações permanece retida em um período de no máximo um minuto após a apresentação do estímulo. Após o período de armazenamento na memória de curto prazo, as informações podem ser esquecidas ou podem passar para o armazenamento na memória de longo prazo, onde podem permanecer retidas por tempo indeterminado e não há limitação quanto ao número de informações a serem retidas, diferentemente da memória de curto prazo que tem capacidade limitada de armazenamento de informações.

A memória de curto prazo também é conhecida como memória operacional ou memória operacional. Segundo Baddeley (1986) este tipo de armazenamento de memória mantêm as informações temporariamente durante a execução de uma determinada tarefa, sendo que esta informação pode ser classificada, organizada e relacionada com outras informações já contidas na memória de longo prazo. Trata-se de um sistema de manipulação temporária da informação que pode auxiliar nos processos de resolução de problemas, leitura, matemática e raciocínio.
De acordo com Baddeley e Hitch (1974), este tipo de memória subdivide-se em três sistemas: o executivo central, a alça fonológica e o esboço visuo-espacial. O executivo central é responsável pelas funções reguladoras, como atenção, controle da ação e resolução de problemas. A alça fonológica é um sistema especializado em reter e manipular informações verbais, assim como o esboço visuo-espacial é um sistema especializado em reter e manipular características físicas dos estímulos, como forma, movimento e cor.

A memória de longo prazo é subdividida em memória explícita ou declarativa e em memória implícita ou não declarativa. Segundo Cohen e Squire (1980) a memória declarativa diz respeito ao "saber que" e a memória não declarativa ao "saber como". Uma memória é considerada explícita ou declarativa quando requer uma recordação consciente para o desenvolvimento de uma determinada tarefa, no entanto, para uma memória ser considerada uma memória implícita ou não declarativa, o desenvolvimento de uma determinada tarefa não requer uma ação consciente (Schacter, 1987), tais como andar de bicicleta, tocar piano e dirigir são exemplos de memórias implícitas (Miotto, Lucia, \& Scaff, 2012).

Ainda, a memória explícita ou declarativa é subdividida em memória episódica e em memória semântica. Segundo Tulving (1972) a memória episódica diz respeito ao armazenamento de acontecimentos pessoais determinados no tempo e no espaço, já a memória semântica refere-se ao armazenamento de conhecimentos gerais sobre o mundo, como fatos, conceitos e vocábulos.

Diante do exposto, o objetivo do presente estudo foi avaliar o impacto do TEPT na memória operacional, na memória visual de curto prazo, na memória episódica de longo prazo, na memória semântica de longo prazo e na memória prospectiva.

\section{Método}

Foram avaliadas vinte mulheres com idades entre 20 e 60 anos, sendo dez mulheres com diagnóstico de TEPT e histórico de sobreposição de situações de violentas (GTEPT). O grupo controle (GC) foi composto pela mesma quantidade de sujeitos que não tinham sido vítimas de violência. Foi realizado o pareamento dos casos a partir do sexo, idade e escolaridade dos sujeitos.

Foram excluídas da amostra mulheres dependentes de substâncias químicas, portadores de esqui- 
zofrenia, portadores de rebaixamento intelectual, portadores de quadros demenciais e de distúrbios neurológicos, pois esses quadros podem interferir na coleta de dados, uma vez que por si só esses quadros já apresentam déficits cognitivos.

A média das idades do GTEPT foi de 41 anos (com desvio-padrão de 13,34) e do GC foi de 41 anos (com desvio-padrão de 13,51). A média de escolaridade de ambos os grupos foi de 12 anos (com desvio-padrão de 2,99).

O diagnóstico de TEPT foi realizado previamente pela equipe médica do Programa de Atendimento e Pesquisa em Violência da Universidade Federal de São Paulo (PROVE), a partir da análise clínica e da aplicação da escala diagnóstica de TEPT, Clinician Administered PTSD Scale (CAPS).

Com relação à medicação no GTEPT, $90 \%$ faziam uso de medicamentos psicoativos, e destes, cerca de $50 \%$ dos sujeitos faziam uso de medicamentos combinados (tomam de um a três tipos de medicações). As medicações utilizadas eram: Ácido Valproico, Escitalopram, Sertralina, Zolpidem, Fluoxetina, Lamotrigina, Nortriptilina, Quetiapina, Topiramato e Risperidona.

No GTEPT, $80 \%$ dos sujeitos sofreram violência física e $20 \%$ dos sujeitos sofreram violência psicológica, sendo que, $100 \%$ destes sujeitos sofreram violências repetidas e/ou na infância. No GC nenhum sujeito sofreu violência recente ou na infância, que possa ter acarretado em um trauma.

Foi utilizado para avaliar a memória o Instrumento de Avaliação Neuropsicológica Breve NEUPSILIN por ser um teste desenvolvido e padronizado recentemente para população brasileira por Fonseca, Salles e Parente (2009). É um teste rápido e de fácil aplicação, com 32 subtestes que avaliam orientação têmporo-espacial, atenção concentrada, percepção visual, habilidades aritméticas, linguagem oral e escrita, memória verbal, praxia e funções executivas. Pode ser aplicado em pessoas de 12 a 90 anos e tem duração de 30 a 50 minutos. Todos os subtestes foram aplicados, porém foram analisados os subtestes que avaliam os processos de memória, alvo do presente estudo, tais como Ordenamento Ascendente de Dígitos, Span Auditivo de Palavras, Memória Episódico-Semântica prova de Evocação Imediata, Memória Episódico-Semântica prova de Evocação Tardia, Memória Episódico-Semântica prova de Reconhecimento, Memória Semântica de Longo Prazo, Memória Visual de Curto Prazo e Memória Prospectiva.

O subteste Ordenamento Ascendente de Dígitos e Span Auditivo de Palavras avaliam memória operacional. Ambos os testes quando somados, seus scores fornecem uma medida geral de memória operacional. Este resultado somado aos scores dos outros subtestes de memória fornecem uma medida de capacidade geral de memória do indivíduo.

No subteste Ordenamento Ascendente de Dígitos o examinador diz uma sequência de números fora de ordem e o examinando fica responsável por repeti-los ao mesmo tempo em que os organiza de modo crescente, ou seja, do menor número para o maior número. A primeira tarefa começa com apenas dois números e posteriormente a quantidade de números e o nível de dificuldade vai aumentando. O número de itens corretos equivale ao escore deste subteste. Este subteste também fornece uma medida de span, que diz respeito a maior quantidade de números em sequência que o sujeito consegue se lembrar durante esta tarefa.

No subteste Span Auditivo de Palavras o examinador lê algumas frases e ao final da leitura destas, o examinando deve repetir as últimas palavras de cada frase. A tarefa se inicia com somente duas frases, depois o nível de complexidade e quantidade de frases vão aumentando. O score deste subteste é calculado da seguinte forma: ganhasse dois pontos por palavra repetida corretamente e na ordem correta e ganhasse um ponto por palavra quando as últimas palavras são lembradas, mas não são descritas na sequência correta. Este subteste também fornece uma medida de span, que diz respeito a maior quantidade de palavras em sequência que o sujeito consegue se lembrar durante esta tarefa.

O subteste Memória Episódico-Semântica é dividido em três provas: Evocação Imediata, Evocação Tardia e Reconhecimento. Na Evocação Imediata o examinador pronuncia uma lista com nove palavras e o examinando tem que repetir as palavras que conseguir se lembrar, não importando a ordem. Na parte de Evocação Tardia o examinador solicita que o examinando diga todas as palavras que ele consiga se lembrar daquela lista apresentada anteriormente, porém existe um intervalo de tempo entre a tarefa de Evocação Imediata e de Evocação Tardia. No Reconhecimento o examinador fala algumas palavras, que incluem palavras da lista e palavras aleatórias, para 
cada palavra o examinando tem que dizer se ela estava na lista apresentada anteriormente ou não. Na Evocação Imediata e na Evocação Tardia, os scores equivalem ao número de palavras recordadas. Na prova de Reconhecimento o score equivale ao número de palavras reconhecidas corretamente somado ao número de distratores negados.

Tanto a Evocação Imediata quanto a Evocação Tardia apresentam uma medida de primazia, recência, intrusões e perseverações. O processo de primazia ocorre quando o examinando se lembra primeiramente das três primeiras palavras da lista e o processo de recência quando se recorda primeiramente das três últimas palavras da lista. Porém os processos de primazia e recência não foram analisados no presente estudo. As intrusões são quaisquer palavras ditas pelos examinandos que não façam parte da lista. As perseverações são quaisquer palavras que foram repetidas mais de uma vez no momento da recordação.

No subteste Memória Semântica de Longo Prazo o examinador faz duas perguntas sobre conhecimentos gerais, sendo que a primeira pergunta vale um ponto e a segunda pergunta vale até quatro pontos. O escore deste subteste é equivalente ao número de respostas corretas.

No subteste Memória Visual de Curto Prazo o examinador mostra uma figura para o examinando durante cinco segundos, em seguida a figura é removida e o examinando fica responsável por reconhece-la dentre três figuras. O número de figuras reconhecidas corretamente é equivalente ao score deste subteste.

No subteste Memória Prospectiva, no início da avaliação o examinador entrega uma folha em branco e solicita que no final da avaliação o examinando deve se lembrar de escrever seu primeiro nome na folha $\mathrm{e}$ entregá-la para o examinador. Após 30 segundos, caso o examinando não se lembre de realizar a tarefa, o examinador pode dar uma dica. Pontua-se com dois pontos quando o examinando se recorda de realizar a tarefa sem a dica e com um ponto quando o examinando se recorda apenas após a dica.

O NEUPSILIN trata-se de um instrumento de rastreio cognitivo, portanto, é composto de tarefas de aplicação rápida e simples. Tem como função a detecção de declínios cognitivos de forma mais abrangente.

No GC foi aplicado um questionário sobre violência antes da aplicação dos instrumentos acima, para assegurar que estes não passaram por situação de violência que possa ter acarretado em trauma. Neste questionário o sujeito assinalava dentre quatro opções, a opção que mais se adequava a ele para cada situação estressora. As opções eram: Aconteceu comigo, Testemunhei, Ouvi dizer e Não aconteceu. Os sujeitos que assinalaram aconteceu comigo ou testemunhei em qualquer uma das situações estressoras foram excluídos da pesquisa. Dentre as situações estressoras presentes no questionário estão: desastre natural, incêndio, explosão, acidente com meio de transporte, grave acidente operacional, grave acidente em casa, grave acidente durante atividade recreativa, exposição a substâncias tóxicas, violência física, violência doméstica, assalto, abuso sexual, outra experiência sexual contra sua vontade, exposição ou combate em zona de guerra, guerrilha urbana, rebelião, sequestro, doença crônica e morte violenta repentina. Ao final do questionário, caso o sujeito acredite que tenha passado por outro tipo de experiência estressante que não tenha sido descrita pelo questionário, ele poderia descrevê-la.

O projeto foi enviado e aprovado pelo CEP-Mackenzie no dia 31 de março de 2014, com Certificado de Apresentação para Apreciação Ética (CAAE) 21697013.3.0000.0084 e número do parecer 575.234. A coleta de dados deu início somente após a aprovação.

O GTEPT foi abordado no PROVE. A avaliação neuropsicológica proposta nesta pesquisa ocorreu antes de qualquer intervenção psicoterápica. O GC foi convidado a participar da pesquisa por meio de cartazes fixados em locais de circulação pública como universidades (após autorização das mesmas), e também foram recrutados sujeitos da comunidade (amostra conveniente).

O projeto se realizou com a cooperação do programa PROVE, para tanto, foi realizada a apresentação formal do projeto e a entrega da Carta de Informação à Instituição, prestando todo e qualquer tipo de esclarecimento que a instituição desejou acerca da pesquisa. A autorização da instituição para a realização da pesquisa foi formalizada através da assinatura do Termo de Consentimento Livre e Esclarecido.

Todos os procedimentos foram explicados de forma clara aos sujeitos. Foi entregue a Carta de Informação ao Sujeito e a aplicação só se iniciou após a assinatura do Termo de Consentimento Livre e Esclarecido, ficando uma cópia com o entrevistado e outra com o entrevistador. Foi informado ao par- 
ticipante que a aplicação poderia ser interrompida a qualquer momento e que caso ele solicitasse a não utilização dos dados, estes seriam descartados. $\mathrm{O}$ anonimato e a confidencialidade dos dados foram garantidos a esses sujeitos.

Foi realizada uma sessão de atendimento para a aplicação do NEUPSILIN com cerca de uma hora de duração. As aplicações ocorreram nas dependências da instituição PROVE ou em local apropriado. O local de aplicação estava bem iluminado, tranquilo e com temperatura adequada. O rapport foi realizado para diminuir a ansiedade do sujeito e para que eles pudessem sentir-se à vontade para a aplicação.

A pesquisa continha riscos mínimos aos participantes e os dados coletados foram utilizados pela instituição PROVE. Estes dados compuseram o prontuário dos pacientes e fizeram parte do atendimento do sujeito na Instituição. Em caso de desconforto ou qualquer outra ocorrência, o voluntário poderia ser atendido pela equipe médica do PROVE.

\section{Resultados}

Para análise dos resultados, as variáveis não numéricas foram analisadas por Qui Quadrado e os resultados obtidos no teste NEUPSILIN foram anali- sados por teste $t$ de Student para amostras dependentes. O nível de significância utilizado foi de $5 \%$.

Os resultados obtidos pelo instrumento NEUPSILIN estão descritos da Tabela 1 à Tabela 4.

Pode-se observar a partir da Tabela 1 que não houve diferença significativa entre os grupos no total dos subtestes de memória $(\mathrm{p}>0,05)$ e no total dos subtestes de memória operacional ( $p>0,05$ ). Por isso, uma análise por subteste foi realizada.

Com relação aos subtestes que avaliam memória operacional, a Tabela 2 mostra que houve diferença significativa entre os grupos no subteste ordenamento ascendente de dígitos $(\mathrm{p}=0,04)$, sendo que $o$ GTEPT teve pior desempenho neste subteste. Porém não houve diferença significativa no subteste Span auditivo de palavras em sentenças ( $p>0,05$ ).

A partir da Tabela 3 podemos notar que não há diferença significativa entre os grupos no que diz respeito ao desempenho de tarefas que envolvam memória episódica.

A Tabela 4 mostra que não há diferença significativa entre os grupos em tarefas que avaliam memória semântica e memória visual, porém pode-se observar diferença significativa entre os grupos em tarefas que envolvam memória prospectiva.

Tabela 1

Valores das médias e desvios-padrões da soma dos subtestes do NEUPSILIN.

\begin{tabular}{lcccc}
\hline Soma & GTEPT & GC & t & p \\
\hline Memória & $47,20(17,35)$ & $53,10(7,69)$ & $-0,98$ & 0,33 \\
Memória operacional & $18,20(11,55)$ & $25,00(4,26)$ & $-1,74$ & 0,09 \\
\hline
\end{tabular}

Nota: Os dados do GTEPT e GC estão descritos em Média (Desvio-Padrão).

Nota: Os dados do GTEPT e GC estão descritos em Média (desvio-padrão).

Tabela 2

Valores das médias e desvios-padrões dos subtestes do NEUPSILIN que avaliam memória de operacional.

\begin{tabular}{lcccc}
\hline Subtestes & GTEPT & GC & $\mathrm{t}$ & $\mathrm{p}$ \\
\hline Ordenamento ascendente de dígitos & $5,50(2,75)$ & $7,50(1,17)$ & $-2,10$ & $0,04^{*}$ \\
Span & $3,70(1,41)$ & $4,20(1,22)$ & $-0,84$ & 0,41 \\
$\begin{array}{l}\text { Span auditivo de palavras em } \\
\text { sentenças }\end{array}$ & $12,70(9,11)$ & $17,50(4,50)$ & $-1,49$ & 0,15 \\
Span & $2,30(1,56)$ & $2,50(0,97)$ & $-0,34$ & 0,73 \\
\hline
\end{tabular}

Nota: Os dados do GTEPT e GC estão descritos acima em Média (Desvio-Padrão); *Coeficientes com diferença significativa $(\mathrm{p}<0,05)$.Nota: Os dados do GTEPT e GC estão descritos acima em Média (desvio-padrão).

*Coeficientes com diferença significativa $(\mathrm{p}<0,05)$. 
Tabela 3

Valores das médias e desvios-padrões dos subtestes do NEUPSILIN que avaliam memória episódica de longo e curto prazo.

\begin{tabular}{lcccc}
\hline Subtestes & GTEPT & GC & $\mathrm{t}$ & $\mathrm{p}$ \\
\hline $\begin{array}{l}\text { Memória verbal } \\
\text { episódico-semântica }\end{array}$ & $5,00(1,63)$ & $4,40(1,50)$ & 0,85 & 0,40 \\
$\begin{array}{l}\text { Evocação Imediata } \\
\quad \text { Intrusões }\end{array}$ & $0,20(0,42)$ & $0,40(0,69)$ & $-0,77$ & 0,44 \\
$\quad \begin{array}{l}\text { Perseverações } \\
\text { Memória verbal }\end{array}$ & $0,00(0,00)$ & $0,10(0,31)$ & $-1,00$ & 0,33 \\
episódico-semântica & & & \\
Evocação Tardia & $2,30(2,45)$ & $2,10(1,44)$ & 0,22 & 0,82 \\
$\quad \begin{array}{l}\text { Intrusões } \\
\quad \text { Perseverações }\end{array}$ & $0,30(0,67)$ & $0,90(0,87)$ & $-1,71$ & 0,10 \\
\hline
\end{tabular}

Nota: Os dados do GTEPT e GC estão descritos acima em Média (desvio-padrão).

Tabela 4

Valores das médias e desvios-padrões dos subtestes do NEUPSILIN que avaliam memória semântica, visual e prospectiva.

\begin{tabular}{lcccc}
\hline Subtestes & GTEPT & GC & $\mathrm{t}$ & $\mathrm{p}$ \\
\hline Memória semântica de longo prazo & $4,70(0,48)$ & $4,80(0,42)$ & $-0,49$ & 0,62 \\
Memória visual de curto prazo & $2,70(0,67)$ & $2,90(0,31)$ & $-0,84$ & 0,40 \\
\hline
\end{tabular}

Nota: Os dados do GTEPT e GC estão descritos acima em Média (desvio-padrão).

*Coeficientes com diferença significativa $(\mathrm{p}<0,05)$.

\section{Considerações finais}

O objetivo deste estudo foi avaliar o impacto do TEPT na memória operacional, na memória visual de curto prazo, na memória episódica de longo prazo, na memória semântica de longo prazo e na memória prospectiva.

A análise aponta para déficits cognitivos em memória operacional e memória prospectiva. Segundo Baddeley (1986), a memória operacional trata-se de um sistema de manipulação temporária da informação que pode auxiliar nos processos de resolução de problemas, leitura, matemática e raciocínio. Já a memória prospectiva, segundo Fonseca, Salles e Parente (2009), seria responsável pela lembrança de intenções futuras, como por exemplo lembrar-se de um compromisso previamente agendado, lembrar de tomar um medicamento, lembrar de comprar algo no supermercado, etc. Tanto a memória operacional, quanto a memória prospectiva utilizam de componentes executivos em seu funcionamento.
Podemos levantar a hipótese de que uma disfunção executiva pode estar acarretando estes déficits cognitivos em memória operacional e em memória prospectiva, e não necessariamente um prejuízo de memória.

De acordo com Shonkoff et al. (2011), a exposição à situações de violência e/ou negligência em períodos precoces podem prejudicar o desenvolvimento das funções executivas. Levando em conta que as mulheres vítimas de violência deste estudo, em sua maioria, sofreram violência na infância, portanto, podemos levantar a hipótese de que este prejuízo em funções executivas pode estar diretamente relacionado a vivência violenta precoce, ocasionando assim, o prejuízo no desenvolvimento executivo.

Peres e Nasello (2005) apontam para diminuição da atividade no córtex pré-frontal em sujeitos com TEPT, o que também corrobora para a hipótese de que os sujeitos com TEPT tenham prejuízos cognitivos relacionados as funções executivas, já que o 
córtex pré-frontal é a área responsável pelo desempenho de tarefas que exijam estas funções.

O córtex pré-frontal é a base dos processos cognitivos complexos e do controle emocional, trata-se de uma área que faz conexões corticais e subcorticais. De acordo com Fuster (2001) o córtex pré-frontal é subdividido em três partes: orbitofrontal, medial e dorsolateral.

A parte orbitofrontal fica responsável pelo controle emocional, a parte medial pelos aspectos motivacionais e a parte dorsolateral pelas funções cognitivas, tais como atenção, memória operacional, planificação, monitorização, flexibilidade mental e categorização (Fuster, 2001).

A capacidade de atenção, não é necessariamente uma função executiva, porém é uma função que contribui significativamente para o desempenho das capacidades executivas (Rebollo, \& Montiel, 2005). A atenção diz respeito a capacidade de concentração para selecionar elementos relevantes, em detrimento de outros (Damásio, 1994). A memória operacional trata-se de um sistema de manipulação temporária da informação (Baddeley, 1986). A planificação é a capacidade para organizar objetivos futuros (Fuster, 2005). A monitorização prepara e controla as ações à medida que elas vão acontecendo (Fuster, 2005). A flexibilidade mental é a capacidade de alternar entre ações e conceitos (Lezak, Howieson, \& Loring 2004). A categorização seria a identificação de características comuns ou diferentes de determinadas situações (Junqué, \& Barroso, 2001).

Os déficits cognitivos associados ao TEPT, como prejuízos na memória operacional e na memória prospectiva encontrados no presente estudo, estão de acordo com a literatura (Stein et al., 2002; Koso, \& Hansen, 2006; Moradi et al., 1999).

No estudo de Stein et al. (2002), no qual foram avaliadas 39 mulheres vítimas de violência, sendo 22 sem diagnóstico de TEPT e 17 com diagnóstico de TEPT, e comparadas com controles saudáveis, observou-se déficits em memória operacional associados ao TEPT.

Koso e Hansen (2006) em seu estudo, também encontraram prejuízos em memória operacional associados ao TEPT, tal estudo foi realizado com a participação de 20 homens veteranos de guerra com diagnóstico de TEPT e 20 homens veteranos de guerra sem diagnóstico de TEPT.
Porém no estudo de Borges (2007), não houveram diferenças significativas entre o grupo meninas vítimas de abuso sexual infantil com diagnóstico de TEPT $(n=12)$ e o grupo meninas controles $(n=16)$, no que diz respeito a memória operacional.

No estudo de Moradi et al. (1999), foram observadas diferenças significativas na memória prospectiva entre o grupo de adolescente e crianças com TEPT $(n=18)$ e o grupo controle $(n=22)$.

Grande parte do GTEPT fazia uso de medicamentos psicoativos, cerca de $90 \%$ do grupo. Todas as medicações utilizadas podem afetar a atenção, entretanto, a medicação Ácido Valpróico pode afetar de maneira global a cognição, porém apenas um dos sujeitos fazia uso deste medicamento (Caetano, 2012).

As alterações cognitivas relacionadas ao TEPT são amplamente discutidas na literatura internacional, porém há poucos artigos brasileiros discutindo esta questão. Um ponto muito importante a ser ressaltado e que difere da literatura estrangeira, é o tipo de violência vivenciado pela população brasileira que desenvolve TEPT. O tipo de violência é bastante divergente dos tipos de violência estudados em artigos estrangeiros, que em sua maioria são sobre homens combatentes de guerra. No Brasil grande parte das vítimas de violência são mulheres e são vítimas de violência urbana, e muitas vezes, podem vivenciar mais que uma situação de violência. Portanto, este artigo torna-se importante por trazer questionamentos sobre o perfil mnemônico deste público tão diferenciado dos estudados em artigos estrangeiros.

Apesar de o presente estudo trazer resultados relevantes quanto ao perfil mnemônico dos sujeitos com diagnóstico de TEPT e sobreposição de violências, seria importante a realização de estudos mais aprofundados sobre o tema. Pois o presente estudo tem limitações quando ao número da amostra, que é consideravelmente pequena, e quanto a dificuldade de encontrar sujeitos com diagnósticos de TEPT que não estejam sendo medicados, pois trata-se de um transtorno com sintomas fisiológicos muito intensos.

Ambas funções cognitivas consideradas como deficitárias neste estudo são de extrema importância para o desempenho de atividades cotidianas. Portanto, a intervenção e reabilitação destas funções no sujeito com TEPT podem auxiliar significativamente na melhora da qualidade de vida. 


\section{Referências}

American Psychiatric Association - APA. (2014). DSM-5: Manual diagnóstico e estatístico de transtornos mentais (5a ed.). Porto Alegre, RS: Artmed.

Atkinson, R. C., \& Shiffrin, R. M. (1968). Human memory: A proposed system and its control processes. In: K. W. Spence, J. T. Spence, Psychology of learning and motivation (Vol. 2, pp. 89-195). New York, NY: Academic.

Baddeley, A. D. (1986). Working memory. Oxford: Clarendon.

Baddeley, A. D. (2000). The episodic buffer: A new component of working memory? Trends in Cognitive Sciences, 4(11), 417-423. https://doi.org/10.1016/S1364-6613(00)01538-2

Baddeley, A. D., \& Hitch, G. J. (1974). Working memory. In: G. Bower (Ed.), The psychology of learning and motivation: Advances in research and theory (Vol. 8, pp. 47-99). New York, NY: Academic.

Borges, J. L. (2007). Abuso sexual e infantil: Consequências cognitivas e emocionais (Dissertação de Mestrado). Universidade Federal do Rio Grande do Sul, Porto Alegre, RS.

Buckley, T. C., Blanchard, E. B., \& Neill, T. (2000). Information processing and PTSD: A review of the empirical literature. Clinical Psychology Review, 28(8), 1041-1065. https://doi.org/10.1016/S0272-7358(99)00030-6

Caetano, N. (2012). Guia de remédios. São Paulo, SP: Escala.

Cerqueira, D., \& Coelho, D. S. C. (2014). Estupro no Brasil: Uma radiografia segundo os dados da Saúde. Brasília, DF: IPEA.

Cloitre, M., Cohen, L. R., Edelman, R. E. \& Han, H. (2001). Posttraumatic stress disorder and extent of trauma exposure as correlates of medical problems and perceived health among women with childhood abuse. Women Health, 34(3),1-17. https://doi.org/10.1016/10.1300/J013v34n03_01

Cohen, N. J. \& Squire, L. R. (1980). Preserved learning and retention of pattern-analyzing skill in amnesia: Dissociation of knowing how and knowing what. Science, 210(4466), 207-210. https://doi.org/10.1126/science.7414331

Damásio, A. (1994). O erro de Descartes. Lisboa: Publicações Europa-América.

Fonseca, R. P., Salles, J. F., \& Parente, M. A. M. P. (2009). Instrumento de avaliação neuropsicológica breve NEUPSILIN. São Paulo, SP: Vetor.

Fuster, J. (2001). The Prefrontal Córtex - an update: Time is of the essence. Neuron, 30(2), 319-333. https://doi. org/10.1016/S0896-6273(01)00285-9

Fuster, J. (2005). Cortex and mind: Unifying cognition. New York, NY: Oxford.

Garcia, L. P. (2013). Violência contra a mulher. Feminicídios no Brasil. Brasília, DF: IPEA.

Glaser, D. (2000). Child abuse and neglect and the brain: A review. Journal of Child Psychology and Psychiatry, 41(1), 97-116.

Graeff, F. G. (2003) Bases biológicas do transtorno de estresse pós-traumático. Revista Brasileira de Psiquiatria, 25(Supl. 1), 21-24.

Heller, S. S., Larrieu, J. A., D’imperio, R., \& Boris, N.W. (1999). Research on resilience to child maltreatment: Empirical considerations. Child Abuse \& Neglect, 23(4), 321-338. https:// doi.org/10.1016/S0145-2134(99)00007-1

Herman, J. L. (1992). Complex PTSD: A syndrome in survivors of prolonged and repeated trauma. Journal of Traumatic Stress, 5(3), 377-391. https://doi.org/10.1002/jts.2490050305

Herman, J., \& Harvey, M. (1997). Adult memories of childhood trauma: A naturalistic clinical study. Journal of Traumatic Stress, 10(4), 557-571. https://doi.org/10.1023/A:1024889601838

Horner, M. D., \& Hamner, M. B. (2002). Neurocognitive functioning in posttraumatic stress disorder. Neuropsychology Review, 12(1), 15-30. https://doi.org/10.1023/A:1015439106231

Junqué, C., \& Barroso, J. (2001). Neuropsicología. Madrid: Síntesis.

Koso, M., \& Hansen, S. (2006). Executive function and memory in posttraumatic stress disorder: A study of Bosnian war veterans. European Psychiatry, 21(3), 167-173. https://doi.org/10.1016/j.eurpsy.2005.06.004

Kristensen, C. H. (2005). Estresse Pós-Traumático: Sintomatologia e funcionamento cognitivo (Tese de Doutorado). Universidade Federal do Rio Grande do Sul, Porto Alegre, RS, Brasil. 
Lezak, M. D., Howieson, D. B., \& Loring, D. (2004). Neuropsychological assessment. (4a ed.). New York, NY: Oxford University.

Lopes, E. M. T., Costa, T. C., Pereira, L. O. L., Fusco, T. M., Oliveira, M. R., et.al. (2015). Violência doméstica e familiar contra a mulher. Brasília, DF: DataSenado.

McEwen, B. (2003). O fim do estresse como nós o conhecemos. Rio de Janeiro, RJ: Nova Fronteira.

Menicucci, E. (2014). Balanço 2014. Brasília, DF: Central de Atendimento á Mulher.

Miotto, E. C., Lucia, M. C. S., \& Scaff, M. (2012). Neuropsicologia clínica. São Paulo, SP: Roca.

Moradi, A. R., Doost, H. T., Taghavi, M. R., Yule, W., \& Dalgleish, T. (1999). Everyday memory deficits in children and adolescents with PTSD: Performance on the Rivermead behavioural memory test. Journal of Child Psychology and Psychiatry, 40(3), 357-361. https://doi.org/10.1111/1469-7610.00453

Pederson, C. L., Maurer, S. H., Kaminski, P. L., Zander, K. A., Peters, C. M., et.al. (2004). Hippocampal volume and memory performance in a community-based sample of women with posttraumatic stress disorder secondary to child abuse. Journal of Traumatic Stress, 17(1), 37-40. https://doi.org/10.1023/B:JOTS.0000014674.84517.46

Peres, J. F. P., \& Nasello, A. G. (2005). Achados da neuroimagem em transtorno de estresse pós-traumático e suas implicações clínicas. Revista de Psiquiatria Clínica, 32(4), 189-201. https://doi.org/10.1590/S0101-60832005000400001

Rebollo, M. A., \& Montiel, S. (2006). Atención y funciones ejecutivas. Revista de Neurologia, 42(Supl. 2), S3-S7.

Roestel, C., \& Kersting, A. (2008) Simple and complex post-traumatic stress disorders: Diagnostic and therapeutic approaches. Der Nervenarzt, 79(7), 845-854. https://doi.org/10.1007/s00115-008-2442-1

Sapolsky, R. M., Romero, L. M., \& Munck, A. U. (2000). How do glucocorticoids influence stress responses? Integrating permissive, suppressive, stimulatory, and preparative actions. Endocrine Reviews, 21(1), 55-89. https://doi. org/10.1210/edrv.21.1.0389

Schacter, D. L. (1987). Implicit memory: History and current status. Journal of Experimental Psychology: Learning, Memory, and Cognition, 13(3), 501-518. https://doi.org/10.1037/0278-7393.13.3.501

Shonkoff, J. P., Duncan, G. J., Fisher, P. A., Magnuson, K., Raver, C. C., et al. (2011). Construindo o sistema de "controle do tráfego aéreo" do cérebro (Fundação Maria Cecília Souto Vidigal, Trad.). Cambridge, MA: Center on the Developing Child at Harvard University.

Stein, M. B., Kennedy, C. M., \& Twamley, E. W. (2002) Neuropsychological function in female victims of intimate partner violence with and without posttraumatic stress disorder. Biological Psychiatry, 52(11), 1079-1088. https:// doi.org/10.1016/S0006-3223(02)01414-2

Tulving, E. (1972). Episodic and semantic memory. In: E. Tulving, W. Donaldson, \& G. H. Bower (Orgs.), The organization of memory. New York, NY: Academic.

Waiselfisz, J. J. (2015). Mapa da violência: Mortes matadas por arma de fogo. Brasília, DF: Flacso-Unesco.

Wignall, E. L., Dickson, J. M., Vaughan, P., Farrow, T. F., Wilkinson, I. D., et.al. (2004). Smaller hippocampal volume in patients with recent-onset posttraumatic stress disorder. Biological Psychiatry, 56(11), 832-836. https://doi. org/10.1016/j.biopsych.2004.09.015

Nathalia Balloni Emygdio

Graduada em Psicologia pela Universidade Presbiteriana Mackenzie, São Paulo-SP, Brasil.

E-mail:naty_nbe@hotmail.com

D https://orcid.org/0000-0002-3104-7835

\section{Simone Freitas Fuso}

Doutora docente no curso de Psicologia da Universidade Presbiteriana Mackenzie, São Paulo-SP, Brasil.

E-mail:simone.fuso@uol.com.br

(iD) https://orcid.org/0000-0001-5014-8650 


\section{Adriana Cristine Fonseca Mozzambani}

Doutora pelo departamento de Psiquiatria e Psicologia Médica da Universidade Federal de São Paulo, São PauloSP, Brasil.

E-mal: a.mozzambani@uol.com.br

(iD) https://orcid.org/0000-0002-4145-0003

Natália Alves Acedo

Graduada em Psicologia pela Universidade Presbiteriana Mackenzie, São Paulo-SP, Brasil.

E-mail:n.alves.acedo@gmail.com

(iD) https://orcid.org/0000-0002-4245-3433

\section{Camila Cruz Rodrigues}

Universidade Presbiteriana Mackenzie. Doutora docente no curso de Psicologia da Universidade Presbiteriana Mackenzie, São Paulo-SP, Brasil.

E-mail: camila.rodrigues@mackenzie.br

(iD https://orcid.org/0000-0003-4116-9569

\section{Marcelo Feijó de Mello}

Livre-Docente pela Escola Paulista de Medicina na Universidade Federal de São Paulo, São Paulo-SP, Brasil.

E-mail:mmello@unifesp.br

(iD) https://orcid.org/0000-0002-0475-4729

Endereço para envio de correspondência:

Universidade Presbiteriana Mackenzie, Centro de Ciências Biológicas e da Saúde. Rua da Consolação, 930, prédio 50. CEP: 01302-907. São Paulo-SP. Brasil.

Recebido 24/01/2017

Aceito $14 / 11 / 2017$

Received $01 / 24 / 2017$

Approved 11/14/2017

Recibido 24/01/2017

Aceptado 14/11/2017

Como citar: Emygdio, N. B., Fuso, S. F., Mozzambani, A. C. F., Acedo, N. A., Rodrigues, C. C., \& Mello, M. F. (2019). Efeitos do transtorno de estresse pós-traumático na memória. Psicologia: Ciência e Profissão, 39, 1-13.

https://doi.org/10.1590/1982-3703003174817

How to cite: Emygdio, N. B., Fuso, S. F., Mozzambani, A. C. F., Acedo, N. A., Rodrigues, C. C., \& Mello, M. F. (2019). Effects of post traumatic stress disorder in Memory. Psicologia: Ciência e Profissão, 39, 1-13.

https://doi.org/10.1590/1982-3703003174817

Cómo citar: Emygdio, N. B., Fuso, S. F, Mozzambani, A. C. F., Acedo, N. A., Rodrigues, C. C., \& Mello, M. F. (2019). Efectos del trastorno de estrés postraumático en la memoria. Psicologia: Ciência e Profissão, 39, 1-13.

https://doi.org/10.1590/1982-3703003174817 